\title{
Sigmoid PID based adaptive safe experimentation dynamics algorithm of portable duodopa pump for Parkinson's disease patients
}

Najwa Hidayah Abdul Razak Ramesh, Mohd Riduwan Ghazali, Mohd Ashraf Ahmad

Faculty of Electrical and Electronics Engineering Technology, University Malaysia Pahang, Pahang, Malaysia

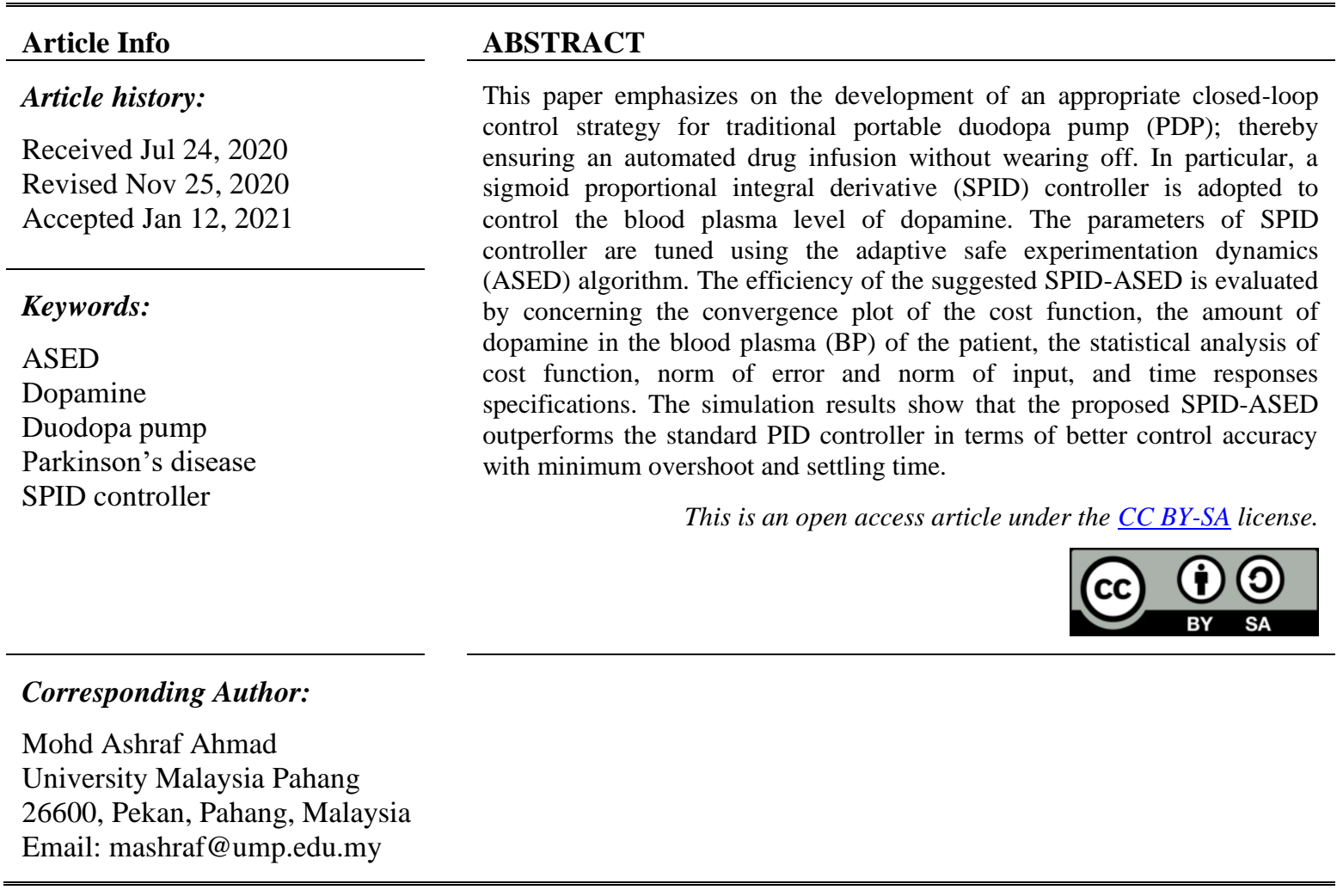

\section{INTRODUCTION}

Parkinson's illness is the most common age-related neurodegenerative disease after Alzheimer's. Today, approximately seven to 10 million individuals around the world have Parkinson's disease (PD) [1, 2]. $\mathrm{PD}$ is caused by the shortfall of nerve cells in the part of the brain called the substantia nigra which responsible for the production of dopamine. In the PD case, if the amount of dopamine produced in the brain starts to decrease or stop, it will cause less or no signal can be transmitted from transmitting neuron to receptor cell of patients in which caused an uncontrolled movement of the patient. Specifically, the movement disorder symptoms start to develop when around $80 \%$ of the nerve cells in the substantia nigra have been lost or when the dopamine level reduced until 20\% [3]. To solve this problem, we can increase the dopamine level by using Levodopa. Levodopa is the best PD medication that passed into the brain and converts it into dopamine [4]. There are two ways to deliver the medicine (Levodopa) which are by using the oral method and the deep brain stimulation (DBS). Nowadays, the most effective method to deliver Levodopa is by using DBS because it can generate more stable plasma and fewer side effects. DBS work in a closed-loop drug infusion with a controller assisted by a controller tuning method to improve the control efficiency and performance of the system. This research important to find the best method to implement in the DBS system to help the patient survive in their daily life without any side effects especially caused by an overdose of Levodopa. By monitoring the level of dopamine in the safe range which is between 10-20 ng/mL for adults. 
Recently, PID-tuned Ziegler-Nichols (ZN) has been utilized for portable duodopa pump system [5]. Here, an ultimate gain $(\mathrm{Ku})$ and oscillation period $(\mathrm{Pu})$ are being used to set the parameters of the PID controller. Although the tuning method based on $\mathrm{ZN}$ is very simple to use, it has many disadvantages, such as further fine-tuning, because the controller settings are aggressive, resulting in the final responses will produce a large overshoot and oscillatory responses. Besides poor performance for processes with a dominant delay, the obtained closed-loop is very sensitive to parameter variations. Therefore, in [5], it is shown that PIDtuned particle swarm optimization (PSO) has been used to overcome the problem of PID-tuned ZN. Note that PSO is an optimization algorithm based on artificial intelligence where particles swarm around in a multidimensional search space to obtain a good solution. Nevertheless, the significant limitation of the PSO proposed in [5] is that the it produces large computational load in obtaining the optimum PID controller parameters. The reason is that the computational load in every iteration is highly depended on the agents' number. Moreover, the existing PID controller structure is still not enough to produce better control accuracy, while it is worth to explore a new modification of PID controller structure to the problem.

Therefore, it encourages us to utilize a trajectory-based optimization since it produces less computational load. To date, there are variety of single agent-based optimization tools such as random search [6], simulated annealing [7], simultaneous perturbation stochastic approximation [8] and safe experimentation dynamics (SED) [9]. From reported work in [10], the adaptive safe experimentation dynamics (ASED) have shown to be the most effective trajectory-based optimization than other trajectorybased optimization tools due to less number of coefficients, memory-based framework (able to keep the best design parameter during the tuning process) and simple algorithm. On the other hand, there are many types of advanced PID have been proposed by many control researchers, such as PD-PID [11], fuzzy-PID [12, 13], PD based fuzzy sliding mode control [14], fractional-PID [15], neural-network-PID (NNPID) [16, 17], fractional-fuzzy-PID [18], kinematic model predictive control PID (K-MPC-PID) [19], integral separation PID (IPID) [20], variable structure PID [21] and also sigmoid-PID (SPID) [22, 23] controllers. Based on the reported advanced PIDs, the SPID is reported to be effective since the proposed method uses variable PID coefficients depending on error signal behaviour according to the sigmoid function. The sigmoid function allows nonlinearly compression of PID controller coefficients between the lower and upper bounds. In other words, the transition between PID boundary parameters takes place asymptotically and continuously through the use of exponential function characteristics. Hence, it is expected that the SPID can produce less overshoot with faster settling time as compared to the PID controller that can lead to better control performance accuracy.

This study proposes a novel controller for a portable duodopa pump (PDP) which Sigmoid-based PID controller. The proposed controller is tuned using the ASED algorithm. Here, the updated controller parameter is improved to adapt to any variation of the cost function during simulation process. The efficacy of the adaptive SED technique is validated to find the optimum SPID control parameters of portable duodopa pump. The control performance of the SPID controller based ASED is evaluated using convergence plot of the cost function, the dopamine level of patient's blood plasma (BP) as well as the quantitative analysis of cost function and its component are shown. Based on the results, it is shown that the SPID controller based ASED could produce a slight enhancement in reducing the error and improve the control accuracy than the conventional PID controller based on the ASED algorithm.

\section{MODELLING OF PORTABLE DUODOPA PUMP}

This section briefly explains the mathematical model of portable duodopa pump taken from [5], which is a modified version of the existing Levodopa dose-effect model [24]. Note that the closed-loop control of dopamine includes the conversion of Levodopa to dopamine which occurs in the brain. This conversion model includes the conversion factor $C$. In particular, the conversion factor $C$ referring to the amount of Levodopa needed to generate $1 \mathrm{ng} / \mathrm{mL}$ of dopamine. Based on [5], the maximum value of Levodopa of a patient after consuming $125 \mathrm{mg}$ capsule (100 $\mathrm{mg}$ of Levodopa and $25 \mathrm{mg}$ of Benserazide) is $3763 \mathrm{ng} / \mathrm{mL}$ and the dopamine range in $\mathrm{BP}$ is $10 \mathrm{ng} / \mathrm{mL}$ to $20 \mathrm{ng} / \mathrm{mL}$ for adults. Let's consider the dopamine level that we want to generate is $15 \mathrm{ng} / \mathrm{mL}$, hence the $C$ is:

$$
C=\frac{1 \mathrm{ng} / \mathrm{mL}}{250.87 \mathrm{ng} / \mathrm{mL}}
$$

Next, the transfer function $G_{P A}(s)$ that relates plasma level of dopamine $M(s)$ and plasma level of Levodopa $N(s)$ is given by:

$$
G_{P A}(s)=\frac{M(s)}{N(s)}=C e^{-\tau_{2} s}
$$


where $\tau_{2}$ (minutes) is referred as recirculation delay. Moreover, the input-output relationships between the amounts of Levodopa infused $O(s)$ and plasma level of dopamine $M(s)$ is modeled as $G_{P K}(s)$ :

$$
G_{P K}(s)=\frac{M(s)}{O(s)}=\frac{C_{P K} e^{-\tau_{1} s}}{\left(1+T_{1} s\right)\left(1+T_{2} s\right)},
$$

where the symbol $C_{P K}$ is the amplification factor while $T_{1}$ and $T_{2}$ are the time constants (minutes), and $\tau_{1}$ is the time lag (minutes) needed to start the absorption of Levodopa from the blood. From (2) and (3), the change in the level of dopamine after we deliver Levodopa is given by:

$$
\Delta D=G_{P K}(s) \times G_{P A}(s)=\frac{C_{P K} e^{-\tau_{1} s}}{\left(1+T_{1} s\right)\left(1+T_{2} s\right)} C e^{-\tau_{2} s},
$$

where $\Delta D=D-D_{o}$. Here, $D_{o}$ is patient body's own dopamine level in BP. There are three stages of $D_{o}$ which are early stage $\left(7<D_{o}<10\right)$, Moderate stage $\left(3<D_{o}<7\right)$ and advanced stage $\left(D_{o}<3\right)$. In this study, the coefficients of the model are $C_{P K}=1418, \tau_{1}=15, \tau_{2}=10, T_{1}=3.2838$ and $T_{2}=36.4380$. Please refer the details of the model in [5] and [25].

\section{METHOD}

This section describes the SPID controller design for portable duodopa pump (PDP) using adaptive safe experimentation dynamics for Parkinson's diseases. Initially, the statement of problem of the SPID controller of a portable duodopa pump is shown. In particular, the SPID controller structure and its control parameters are described. Then, the step-by-step guideline is presented to obtain the optimum SPID parameters using ASED.

\subsection{Statement of problem}

Figure 1 depicts the SPID control system block diagram for a portable duodopa pump. The symbols $D, r(t), e(t), z(t)$, and $y(t)$ are denoted as portable duodopa pump system, reference amount dopamine [5], error signal, control input and level of dopamine response, respectively. Note that, the system $D$ is obtained from (4), where $D=\Delta D+D_{o}$.

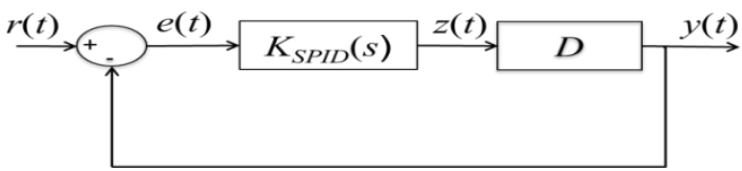

Figure 1. Block diagram of control system for portable duodopa pump

Moreover, the SPID controller $K_{S P I D}(s)$ is given by:

$$
K_{S P I D}(s)=K_{p v}+\frac{K_{i v}}{s}+K_{d v} s
$$

where,

$$
\begin{aligned}
& K_{p v}=K_{\text {plow }}-\left|\frac{K_{\text {phigh }}-K_{\text {plow }}}{1+e^{-\alpha_{p}|e(t)|}}\right|, \\
& K_{\text {iv }}=K_{\text {ilow }}-\left|\frac{K_{\text {ihigh }}-K_{\text {ilow }}}{1+e^{-\alpha_{i}|e(t)|}}\right|, \\
& K_{d v}=K_{\text {dlow }}-\left|\frac{K_{\text {dhigh }}-K_{\text {dlow }}}{1+e^{-\alpha_{d}|e(t)|}}\right| .
\end{aligned}
$$

Note that the (6)-(8) are formulated based on sigmoid function [22]. One of the important advantages of the proposed solution is that the sigmoid function ensures a continuous change of the SPID parameters according to the change of error $e(t)$. For simplicity of the SPID controller design, we introduce $\Delta_{p}=K_{\text {phigh }}-K_{\text {plow }}, \Delta_{i}=K_{\text {ihigh }}-K_{\text {ilow }}$ and $\Delta_{d}=K_{\text {dhigh }}-K_{\text {dlow }}$. 
The purpose of the controller for portable duodopa pump is to make sure the optimal delivery of Levodopa to a patient based on the patient requirement with fast response and less overshoot of dopamine level. In addition, the level of levodopa infused by the controller is increasing proportionally to the reduction in the plasma level of dopamine [5]. Therefore, the cost function for this problem is set as (9):

$$
J(K, \Delta, \alpha)=w_{1} \bar{E}+w_{2} \bar{Z},
$$

Where

$$
\begin{aligned}
& \bar{E}=\int_{t_{0}}^{t_{f}}|e(t)|^{2} d t, \\
& \bar{Z}=\int_{t_{0}}^{t_{f}}|z(t)|^{2} d t,
\end{aligned}
$$

for the time duration of $\left[t_{0}, t_{f}\right]$. The symbols $t_{0}$ and $t_{f}$ are refers to the initial and final time, respectively. Based on (9), the symbols $K=\left[\begin{array}{lll}K_{\text {plow }} & K_{\text {ilow }} & K_{\text {dlow }}\end{array}\right]^{\mathrm{T}}, \Delta=\left[\begin{array}{lll}\Delta_{p} & \Delta_{i} & \Delta_{d}\end{array}\right]^{\mathrm{T}}$, and $\alpha=\left[\begin{array}{lll}\alpha_{p} & \alpha_{i} & \alpha_{d}\end{array}\right]^{\mathrm{T}}$ that bring to 9 tuning parameters of SPID controller. Note that $\bar{E}$ and $\bar{Z}$ can be also defined as norm of error and norm of input, respectively. Next, there are two weight coefficients adopted in (9), which are represented by the symbols $w_{1} \in \mathbf{R}$ and $w_{2} \in \mathbf{R}$ to prioritize the performance index in the multi-cost function. Finally, our problem can be stated as:

Problem 1. From the plant $D$ in Figure 1 and the measured output $y(t)$ and input $z(t)$ data, obtain the SPID control parameters in $K_{\text {SPID }}(s)$ such that the cost function in (9) is reduced.

\subsection{SPID utilizing adaptive safe experimentation dynamics}

The implementation of ASED method to obtain the optimum SPID controller of portable duodopa pump system is explained in this section. Initially, the conventional SED algorithm is briefly described. Then, the ASED method with its procedure, is shown. Finally, a complete step-by-step guideline of ASED method to obtain the optimum SPID controller of portable duodopa pump is presented.

Next, let the optimization problem given as (12):

$$
\min _{\boldsymbol{v} \in \mathrm{R}^{n}} g(\boldsymbol{v})
$$

where $g: \mathbf{R}^{n} \rightarrow \mathbf{R}$ is a function with the vector of design parameter $\boldsymbol{v} \in \mathbf{R}^{n}$. The SED method updates $\boldsymbol{v} \in \mathbf{R}^{n}$ to obtain an optimum solution $\overline{\boldsymbol{v}} \in \mathbf{R}^{n}$ of (12) by using:

$$
\boldsymbol{v}_{i}(k+1)= \begin{cases}h\left(\overline{\boldsymbol{v}}_{i}-K_{g} r_{1}\right) & \text { if } r_{1} \leq E \\ \overline{\boldsymbol{v}}_{i} & \text { if } r_{1}>E\end{cases}
$$

where $k=0,1, \ldots$, is number of iteration, $\boldsymbol{v}_{i} \in \mathbf{R}$ is the $i$ th component of $\boldsymbol{v} \in \mathbf{R}^{n}$, and $\overline{\boldsymbol{v}}_{i} \in \mathbf{R}$ is the $i$ th elements of current best vector $\bar{v} \in \mathbf{R}^{n}$. The coefficient $K_{g}$ is the size of the interval to discover the random steps on $\boldsymbol{v}_{i} \in \mathbf{R}$, the coefficient $E$ is the probability to change the element of the tuning parameter and $r_{1} \in \mathbf{R}$ is the random value that is produced uniformly between 0 and 1 . The function $h$ in (13) is expressed by:

$$
h(.)=\left\{\begin{array}{rlc}
\boldsymbol{v}_{\max } & \text { if } & \overline{\boldsymbol{v}}_{i}-K_{g} r_{2}>\boldsymbol{v}_{\max }, \\
\overline{\boldsymbol{v}}_{i}-K_{g} r_{2} & \text { if } & \boldsymbol{v}_{\min } \leq \overline{\boldsymbol{v}}_{i}-K_{g} r_{2} \leq \boldsymbol{v}_{\max } \\
\boldsymbol{v}_{\min } & \text { if } & \overline{\boldsymbol{v}}_{i}-K_{g} r_{2}<\boldsymbol{v}_{\min }
\end{array}\right.
$$

where $\boldsymbol{v}_{\min }$ and $\boldsymbol{v}_{\max }$ are the pre-defined upper and lower bounds, respectively, and $r_{2}$ is another random value that is produced independently from $r_{1}$, in the range of $[0,1]$. The procedures for the execution of the SED algorithm are given by:

Step 1: Obtain the $\boldsymbol{v}_{\text {min }}, \boldsymbol{v}_{\text {max }}, K_{g}$ and $E$ values and set the initial design parameter $\boldsymbol{v}(0)$. Evaluate the cost function $g(\boldsymbol{v}(0))$ and set $\overline{\boldsymbol{v}}=\boldsymbol{v}(0)$ and $\bar{g}=g(\boldsymbol{v}(0))$.

Step 2: Execute $\overline{\boldsymbol{v}}=\boldsymbol{v}(k)$ and $\bar{g}=g(\boldsymbol{v}(k))$ when the value $g(\boldsymbol{v}(k))<\bar{g}$ otherwise, go to Step 3.

Step 3: Produce random values $r_{1}$ and $r_{2}$ and obtain the updated equation in (13).

Step 4: Execute the cost function $g\left(\boldsymbol{v}_{i}(k+1)\right)$. 
Step 5: If the maximum number of iteration $k_{\max }$ is achieved, the algorithm stop with the optimum parameter $\bar{v}:=\arg \min _{\boldsymbol{v} \in\{\boldsymbol{v}(0), \boldsymbol{v}(1), \ldots, \boldsymbol{v}(k+1)\}} g(\boldsymbol{v})$. Otherwise, repeat Step 2.

Based on the study from [10], the conventional SED algorithms are still insufficient to produce a significant convergence accuracy. One of the reason is that the design parameters updated in (13) depend only on $K_{g}$ and the random numbers $r_{1}$ and $r_{2}$, which makes it identical to the basic random search strategy. Therefore, in order to solve the above issue, we adopt the Adaptive SED from [10] as the optimization tool in finding the optimal SPID controller. In particular, the updated in (13) is modified as (15):

$$
\boldsymbol{v}_{i}(k+1)= \begin{cases}h\left(\overline{\boldsymbol{v}}_{i}-K_{g} r_{2}\right)+K_{g 1}\left(\frac{g(\boldsymbol{v}(k))-\bar{g}}{g(\boldsymbol{v}(k))}\right) & \text { if } r_{1} \leq E, \\ \overline{\boldsymbol{v}}_{i}+K_{g 1}\left(\frac{g(\boldsymbol{v}(k)-\bar{g})}{g(\boldsymbol{v}(k))}\right) & \text { if } r_{1}>E,\end{cases}
$$

where $K_{g 1}$ is a new scalar value that defines the size of the interval to adjust the adaptive mechanism. Note that, by adopting (15), the probability of perturbing each element of the tuning parameter is increased, which could lead to the extra effort of finding the optimal tuning parameter [10]. described as:

Finally, the procedure to execute the ASED for SPID control tuning of portable duodopa pump is

Procedure 1: Set the maximum iterations $k_{\max }$ and consider $g(\boldsymbol{v})=J(K, \Delta, \alpha)$ and $\boldsymbol{v}_{i}=\log \psi_{i}$. Note that $\psi=\left[\begin{array}{lllllllll}K_{\text {plow }} & \Delta_{p} & \alpha_{p} & K_{\text {ilow }} & \Delta_{i} & \alpha_{i} & K_{\text {dlow }} & \Delta_{d} & \alpha_{d}\end{array}\right]^{\mathrm{T}}$ is the tuning parameter vector and $\psi_{i}=10^{v_{i}}(i=$ $1,2, \ldots, 9)$.

Procedure 2: Execute the ASED algorithm in (15).

Procedure 3: After $k_{\max }$ is obtained, record the optimal tuning parameter $\overline{\boldsymbol{v}}$. Finally, execute the SPID controller $\psi=\left[\begin{array}{llll}10^{\bar{v}_{1}} & 10^{\bar{v}_{2}} & \cdots & 10^{\bar{v}_{9}}\end{array}\right]^{\mathrm{T}}$ in Figure 1

\section{RESULTS AND DISCUSSIONS}

The efficiency of the ASED based method in obtaining the optimum value of SPID controller is presented in this section. Specifically, the convergence plot response of the cost function, the dopamine level in patient's BP and the statistical analysis of cost function, norm of error and norm of input are demonstrated and analyzed. Here, 30 trials are utilized to assess the statistical performances of SPID based on ASED. The simulation is executed using the reference $r(t)$ :

$$
r(t)=(15 \mathrm{ng} / \mathrm{mL}) \text {, for } 0<t<900 \text { minutes }
$$

The aim is to obtain the optimum SPID control parameters such that the dopamine sensor can follow the pre-defined reference in (16) with fast response and minimum overshoot. The coefficients of ASED for tuning SPID are given as $K_{g}=0.08, E=0.63, v_{\min }=0.003, \boldsymbol{v}_{\max }=20$ and $K_{g 1}=0.001$, while for tuning PID, the coefficients of ASED are given as $K_{g}=0.002, E=0.66, \boldsymbol{v}_{\min }=0, \boldsymbol{v}_{\max }=3$ and $K_{g 1}=0.0002$. The weighting coefficients for tuning both SPID and PID are set as $w_{1}=100$ and $w_{2}=1$. The initial values of the tuning parameters are given as $\boldsymbol{v}(0)=\left[\begin{array}{lllllllllll}0.14571 & 0 & 0 & 0.00589 & 0 & 0 & 0.00442 & 0 & 0\end{array}\right]^{\mathrm{T}}$. Note that the initial values are taken from the value of PID controller tuned by Ziegler Nichols in [5] by guaranteeing a stable response is obtained.

Figure 2 illustrates the response of the cost function convergence plot of SPID controller tuning

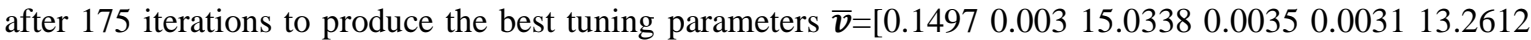
$0.00300 .003115 .8531]^{\mathrm{T}}$. Note that the convergence response in Figure 2 is the best convergence response selected from 30 trials. It proves that the ASED based method can reduce the cost function and produce better control accuracy, as shown by the dopamine level response in Figure 3. Here, three of dash lines in Figure 3 correspond to the responses of the controller based on initial PID parameters or PID tuned by ZN $(k=0)$, while three solid lines refer to optimal PID parameters $(k=175)$ and the other three dash-dot lines are referred to optimal SPID parameters $(k=175)$. Three different colors are used to distinguish between each stage of PD patients which are, black for early stage, blue for Moderate stage and purple indicates advanced stage of PD patients' response. It shows that the SPID based ASED successfully achieves to improve the performance of the duodopa pump at every stage of Parkinson's disease. When we focus on the early stage of PD patient output response which was plotted in Figure 3, the SPID-ASED produced very minimal overshoot with a peak value of $15.6117 \mathrm{ng} / \mathrm{mL}$ as compared to the PID-ASED and the PID-ZN with $15.7933 \mathrm{ng} / \mathrm{mL}$ and $18.4300 \mathrm{ng} / \mathrm{mL}$, respectively. Moreover, the SPID-ASED also produces slightly faster settling time with a 
value of 124.4099 minutes than the PID-ASED and PID-ZN with values of 134.1324 minutes and 294.9286 minutes, respectively. A similar pattern can be seen for other stages of PD patient. It proves that SPID based ASED tuning method has improved performance control accuracy compared to PID-ASED and PID-ZN controllers.

Furthermore, Table 1 shows the comparative assessments for both PID-ASED and SPID-ASED controllers after 30 trials, in terms of the cost function, norm of error, norm of input, and time response specifications. Note that the data in Table 1 is taken for the case of PD patients at an early stage. In particular, the bold numerical values in Table 1 imply the best performance. Moreover, it demonstrates that the SPIDASED produces slightly better statistical values (in terms of mean, best, and worst) in the cost function and norm of error than the PID-ASED. Thence, it proves that the proposed SPID based ASED can produce enhancement in the control accuracy, especially on the cost function and the tracking error.

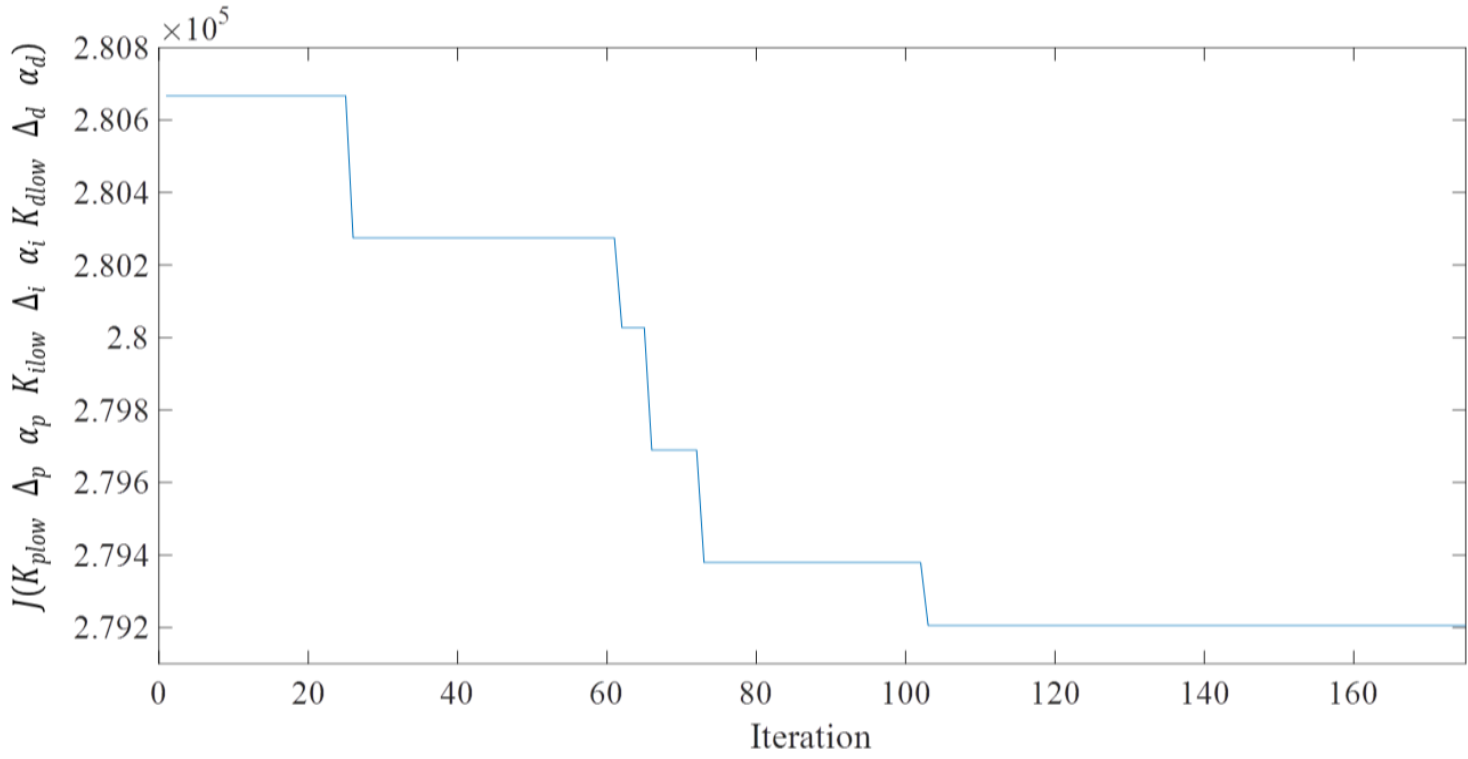

Figure 2. Convergence plot of the cost function of SPID-ASED

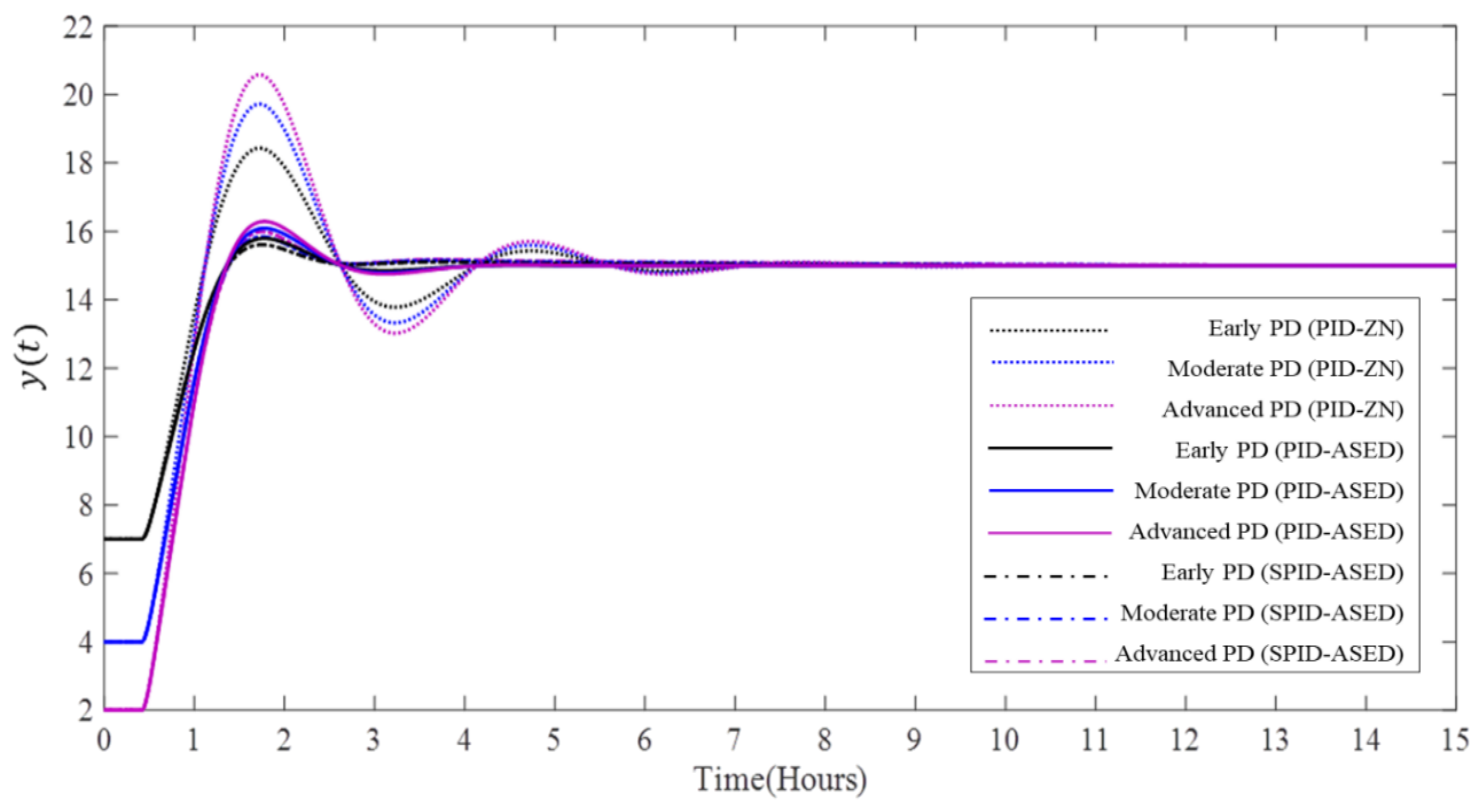

Figure 3. Blood plasma level of dopamine for intra-patient variability 
Table 1. Statistical performances between SPID-ASED and PID-ASED controllers.

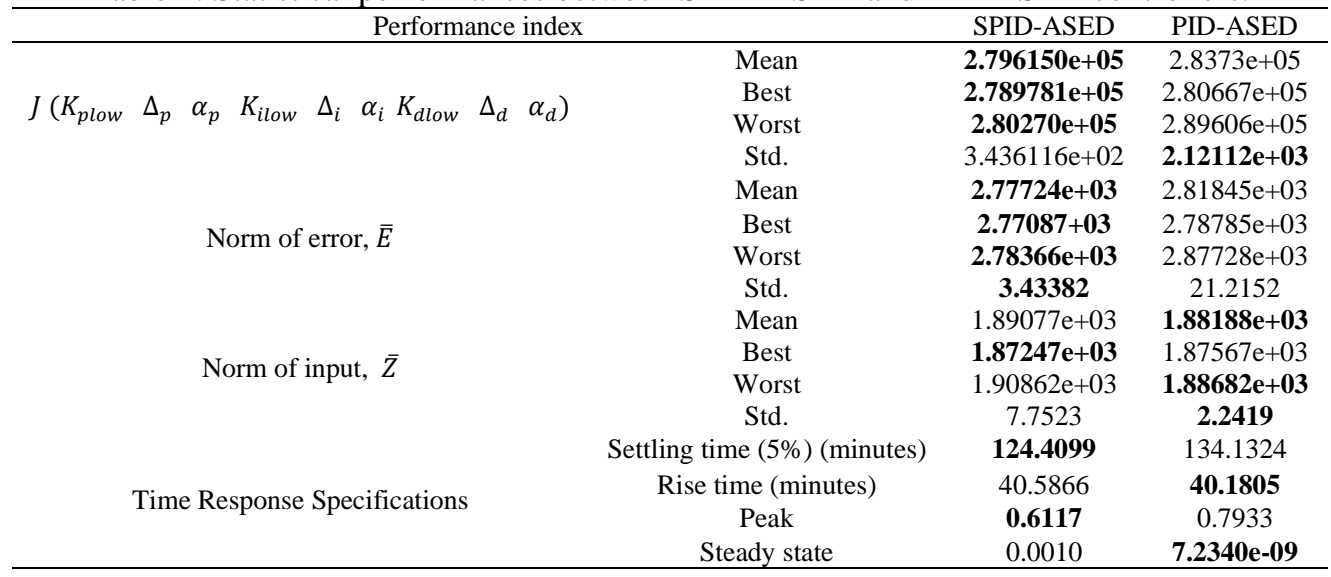

\section{CONCLUSION}

The SPID controller using the ASED method for a portable duodopa pump system has been presented. Simulation results have shown that the ASED-tuned SPID controller has enhanced the control accuracy as it successfully improves duodopa pump performance at each PD stage by providing a better optimal solution compared to PID-ASED. In particular, early stage statistical analyses of PD patients revealed that the proposed SPID controller is able to generate lower cost function values, norm of error and norm of input compared to the PID controller. The findings are supported by the response of the blood plasma level of dopamine to intra-patient variability. In the future, the SPID-ASED controller can be extended for controlling various medical devices, such as a continuous positive airway pressure ventilator and Neuromodulator devices.

\section{ACKNOWLEDGEMENTS}

The financial support of the project is supported by UMP internal grant RDU1803137.

\section{REFERENCES}

[1] E. Naqvi, "Levodopa," Parkinson's News Today, 02-Apr-2019. [Online]. Available at: https://parkinsonsnewstoday.com/levodopa/. [Accessed: 14-Apr-2020].

[2] E. Naqvi, "Parkinson's disease statistics," Parkinson's News Today, 06-Aug-2018. [Online]. Available at: https://parkinsonsnewstoday.com/parkinsons-disease-statistics/. [Accessed: 14-Apr-2020].

[3] M. C. Rodriguez-Oroz, M. Jahanshah, P. Krack, I. Litvan, R. Macias, E. Bezard, and J. A. Obeso, "Initial clinical manifestations of Parkinsons disease: features and pathophysiological mechanisms," The Lancet Neurology, vol. 8, no. 12, pp. 1128-1139, 2009.

[4] M. Contin and P. Martinelli, "Pharmacokinetics of levodopa," Journal of Neurology, vol. 257, no. 2, pp. 253-261, 2010.

[5] Pravika M., J. Jacob, and Paul J. K., "PID controlled fully automated portable duodopa pump for Parkinson's disease patients," Biomedical Signal Processing and Control, vol. 50, pp. 178-187, 2019.

[6] Y. Shang and J. Chu, "A method based on random search algorithm for unequal circle packing problem," International Conference on Information Science and Cloud Computing Companion, pp. 43-47, 2013.

[7] L. F. Fraga-Gonzalez, R. Q. Fuentes-Aguilar, A. Garcia-Gonzalez, and G. Sanchez-Ante, "Adaptive simulated annealing for tuning PID controllers," AI Communications, vol. 30, no. 5, pp.347-362, 2017.

[8] M. A. Ahmad, S.-I. Azuma, and T. Sugie, "Performance analysis of model-free PID tuning of MIMO systems based on simultaneous perturbation stochastic approximation," Expert Systems with Applications, vol. 41, no. 14, pp. 6361-6370, 2014.

[9] N. S. A. Shukor, M. A. Ahmad, and M. Z. M. Tumari, "Data-driven PID tuning based on safe experimentation dynamics for control of liquid slosh," IEEE 8th Control and System Graduate Research Colloquium (ICSGRC), pp. 62-66, 2017.

[10] M. R. B. Ghazali, M. A. B. Ahmad, and R. M. Taufika Bin Raja Ismail, "Adaptive safe experimentation dynamics for data-driven neuroendocrine-PID control of MIMO systems," IETE Journal of Research, pp. 1-14, 2019.

[11] A. N. K. Nasir, R. M. T. Raja Ismail, and M. A. Ahmad, "Performance comparison between sliding mode control (SMC) and PD-PID controllers for a nonlinear inverted pendulum system," Conference: International Conference Control, Automation, Robotics and Vision, pp. 122-127, 2010.

[12] J. S. Glower and J. Munighan, "Designing fuzzy controllers from a variable structures standpoint," IEEE Transactions on Fuzzy Systems, vol. 5, no. 1, pp. 138-144, Feb. 1997. 
[13] T. Hiyama, M. Kugimiya, and H. Satoh, "Advanced PID type fuzzy logic power system stabilizer," IEEE Transactions on Energy Conversion, vol. 9, no. 3, pp. 514-520, Sept. 1994.

[14] L. Teng, M. A. Gull and S. Bai, "PD based fuzzy sliding mode control of a wheelchair exoskeleton robot," IEEE/ASME Transactions on Mechatronics, 2020.

[15] C. Yeroglu and A. Ates, "A stochastic multi-parameters divergence method for online auto-tuning of fractional order PID controllers," Journal of the Franklin Institute, vol. 351, no. 5, pp. 2411-2429, 2014.

[16] J. Wang, C. Zhang, Y. Jing, and D. An, "Study of neural network PID control in variable-frequency airconditioning system," 2007 IEEE International Conference on Control and Automation, pp. 317-322, 2007.

[17] J. Liu, M. Liu, D. Pei, and H. Sun, "FPGA implementation of family service robot based on neural network PID motion control system," 2019 International Conference on Electronic Engineering and Informatics (EEI), pp. 304308, 2019.

[18] R. Bhimte, K. Bhole, and P. Shah, "Fractional order fuzzy PID controller for a rotary servo system," 2018 2nd International Conference on Trends in Electronics and Informatics (ICOEI), pp. 538-542, 2018.

[19] L. Tang, F. Yan, B. Zou, K. Wang, and C. Lv, "An improved kinematic model predictive control for high-speed path tracking of autonomous vehicles," IEEE Access, vol. 8, pp. 51400-51413, 2020.

[20] L. Jia and X. Zhao, "An improved particle swarm optimization (PSO) optimized integral separation PID and its application on central position control system," IEEE Sensors Journal, vol. 19, no. 16, pp. 7064-7071, 2019.

[21] I. Boiko, X. Sun, and E. Tamayo, "Performance analysis and tuning of variable-structure PID controllers for level process," 2009 IEEE Control Applications, (CCA) \& Intelligent Control, (ISIC), pp. 268-273, 2009.

[22] A. Ateş, B. B. Alagöz, C. Yeroğlu, and H. Alisoy, "Sigmoid based PID controller implementation for rotor control," 2015 European Control Conference (ECC), pp. 458-463, 2015.

[23] M. A. Ahmad and R. M. T. R. Ismail, "A data-driven sigmoid-based PI controller for buck-converter powered DC motor," 2017 IEEE Symposium on Computer Applications \& Industrial Electronics (ISCAIE), pp. 81-86, 2017.

[24] S. S. Hacisalihzade, M. Mansour, C. Albani, and G. Baumgartner, "Optimal control of parkinsonian symptoms," 26th IEEE Conference on Decision and Control, pp. 2027-2030, 1987.

[25] S. S. Hacisalihzade, M. Mansour, and C. Albani, "Optimization of symptomatic therapy in Parkinson's disease," IEEE Transactions on Biomedical Engineering, vol. 36, no. 3, pp. 363-372, March 1989. 